Submitted to Astrophysical Journal Letters

\title{
Strong Clustering of High-Redshift Lyman-alpha Forest Absorption Systems
}

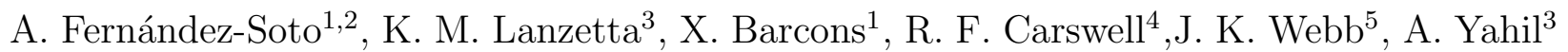

\begin{abstract}
We use new observations of very weak CIV absorption lines associated with high-redshift Ly $\alpha$ absorption systems to measure the high-redshift Ly $\alpha$ line two-point correlation function (TPCF). These very weak CIV absorption lines trace small-scale velocity structure that cannot be resolved by Ly $\alpha$ absorption lines. We find that (1) high-redshift Ly $\alpha$ absorption systems with $N(\mathrm{H} \mathrm{I})>3 \times 10^{14} \mathrm{~cm}^{-2}$ are strongly clustered in redshift, (2) previous measurements of the Ly $\alpha$ line TPCF underestimated the actual clustering of the absorbers due to unresolved blending of overlapping velocity components, (3) the present observations are consistent with the hypothesis that clustering of Ly $\alpha$ absorption systems extends to lower column densities, but maybe with smaller amplitude in the correlation function, and (4) the observed clustering is broadly compatible with that expected for galaxies at $z \sim 2-3$. We interpret these results as suggesting that many or most Ly $\alpha$ absorbers may arise in galaxies even at high redshifts, and, therefore, that the Ly $\alpha$ forest probes processes of galaxy formation and evolution for redshifts $z \lesssim 5$.
\end{abstract}

Subject headings: quasars: absorption lines, galaxies: evolution

\footnotetext{
${ }^{1}$ Instituto de Física de Cantabria, Consejo Superior de Investigaciones Científicas - Universidad de Cantabria, Facultad de Ciencias, 39005 Santander, SPAIN.

${ }^{2}$ Departamento de Física Moderna, Universidad de Cantabria, 39005 Santander, SPAIN.

${ }^{3}$ Astronomy Program, Department of Earth and Space Sciences, State University of New York at Stony Brook, Stony Brook, NY 11794-2100, U.S.A.

${ }^{4}$ Institute of Astronomy, University of Cambridge, Cambridge CB3 0HA, U.K.

${ }^{5}$ School of Physics, University of New South Wales, P.O. Box 1, Kensington, NSW, AUSTRALIA
} 


\section{Introduction}

The observational result that high-redshift Ly $\alpha$ absorption systems appear not to cluster strongly in redshift (e.g. Sargent et al. 1980) has driven most discussion about the origin of the Ly $\alpha$ forest. This result has generally been interpreted as evidence that high-redshift Ly $\alpha$ absorbers arise in intergalactic clouds rather than in galaxies. Recent studies of the relationship between Ly $\alpha$ absorbers and galaxies at redshifts $z \lesssim 1$, however, directly demonstrate that many or most low-redshift Ly $\alpha$ absorbers (or at least those

satisfying $W_{\text {rest }}(\operatorname{Ly} \alpha) \gtrsim 0.3 \AA$ ) arise in galaxies rather than in intergalactic clouds (Lanzetta et al. 1995). Why is it that Ly $\alpha$ absorbers appear not to cluster strongly in redshift whereas low-redshift Ly $\alpha$ absorbers appear to arise in galaxies?

One suggestion is that there exist two distinct populations of Ly $\alpha$ absorbers: a rapidly evolving, unclustered, intergalactic population that dominates at high redshifts, and a slowly evolving, clustered, galactic population that dominates at low redshifts (e.g. Bahcall et al. 1995). Another possibility is that previous measurements of the high-redshift Ly $\alpha$ two-point correlation function (TPCF) have underestimated the actual clustering of the absorbers - presumably due to unresolved blending of overlapping velocity components - and Ly $\alpha$ absorbers arise in galaxies at all epochs.

Here we examine the second of these possibilities, that previous measurements of the high-redshift Ly $\alpha$ TPCF have underestimated the actual clustering of the absorbers, using new observations of very weak CIV absorption lines associated with high-redshift Ly $\alpha$ absorbers (Cowie et al. 1995, hereafter CSKH), §2. These very weak CIV absorption lines trace small-scale velocity structure that cannot be resolved by Ly $\alpha$ absorption lines because (1) the atomic weight of $\mathrm{C}$ is 12 times the one of $\mathrm{H}$, so the thermal broadening of CIV absorption lines is 3.5 times smaller than that of Ly $\alpha$ lines, and (2) CIV absorption lines suffer far less saturation because of the difference in column densities. We show that the CIV lines indeed help to reveal the underlying velocity correlation of the Ly $\alpha$ systems, and that this same velocity structure is blended away in the Ly $\alpha$ data, §3. We conclude with a comparison of the derived velocity clustering of the Ly $\alpha$ absorbers with that of galaxies at the present epoch, $§ 4$.

\section{Data}

The observations by CSKH consist of high spectral resolution (FWHM $\approx 8 \mathrm{~km} \mathrm{~s}^{-1}$ ), high signal-to-noise ratio $(S / N \approx 50$ per resolution element) spectra of three QSOs obtained with the Keck telescope and the HIRES spectrograph. The observations generally cover 
both the Ly $\alpha$ and the corresponding CIV wavelength regions and are sensitive to CIV absorption lines arising in CIV column densities as low as $N(\mathrm{CIV}) \approx 10^{12} \mathrm{~cm}^{-2}$.

From the observations, CSKH selected a complete sample of 38 Ly $\alpha$ absorption lines satisfying $N(\mathrm{H} \mathrm{I}) \geq 3 \times 10^{14} \mathrm{~cm}^{-2}$. They then eliminated seven of these absorption lines due to contamination by unrelated metal absorption lines or lack of coverage of the corresponding CIV wavelength region or because the lines produce corresponding Lyman-limit absorption (which indicates $N(\mathrm{H} \mathrm{I}) \gtrsim 2 \times 10^{17} \mathrm{~cm}^{-2}$ ). The resulting sample thus contains 31 Ly $\alpha$ absorption lines satisfying $3 \times 10^{14} \mathrm{~cm}^{-2} \leq N(\mathrm{H} \mathrm{I}) \leq 2 \times 10^{17} \mathrm{~cm}^{-2}$. For each member of this sample, they searched the corresponding CIV wavelength region for CIV absorption lines and applied a Voigt profile fitting procedure to the identified CIV absorption lines to measure redshifts, Doppler parameters, and column densities.

Here we use the absorption system parameters derived by CSKH in their profile analysis, which are summarized in their Table 1a. The average redshift of the absorbers is $\langle z\rangle=2.6$, the median column density of the absorbers is $N(\mathrm{H} \mathrm{I})=8.1 \times 10^{14} \mathrm{~cm}^{-2}$, and the typical CIV/HI ratio of the absorbers is $3 \times 10^{-3}$. Of the final sample of 31 Ly $\alpha$ absorption lines, 15 are observed to have associated CIV absorption, of which six show small-scale velocity structure with between two and nine velocity components per Ly $\alpha$ absorption line.

\section{Analysis}

\subsection{High-Redshift Ly $\alpha$ Two-point Correlation Function}

Our primary assumption is that very weak CIV absorption lines trace small-scale velocity structure that cannot be resolved by Ly $\alpha$ absorption lines. Hence the goal of the analysis is to measure the high-redshift Ly $\alpha$ TPCF by using very weak CIV absorption lines instead of the Ly $\alpha$ absorption lines themselves.

To do this we use the results summarized in Table 1a of CSKH. In cases where CSKH identified one or more CIV absorption lines with a single Ly $\alpha$ absorption line, we use the redshifts of all CIV absorption lines in the analysis. In cases where CSKH identified no CIV absorption lines with a single Ly $\alpha$ absorption line, we use the single redshift of the Ly $\alpha$ absorption line in the analysis. This procedure yields a total of 52 absorption redshifts. We then use these absorption redshifts to construct the Ly $\alpha$ line TPCF by normalizing the distribution of velocity pairs with respect to an unclustered distribution of redshifts.

The results are shown in Figure 1, which plots in the upper panel the high-redshift Ly $\alpha$ line TPCF as traced by CIV absorption lines. (The error bars shown in Figure 1 are 
based on a modified "bootstrap" technique that yields approximately correct results even for correlated data. Details of this technique will be presented elsewhere.) It is clear from Figure 1 that the high-redshift Ly $\alpha$ TPCF indicates very strong clustering on velocity scales $\lesssim 250 \mathrm{~km} \mathrm{~s}^{-1}$. We therefore conclude that high-redshift Ly $\alpha$ absorption systems with $N(\mathrm{H} \mathrm{I})>3 \times 10^{14} \mathrm{~cm}^{-2}$ are strongly clustered in redshift.

\subsection{Blending of Overlapping Velocity Components}

The results of $\S 3.1$ demonstrate that high-redshift Ly $\alpha$ absorption systems with $N(\mathrm{H} \mathrm{I})>3 \times 10^{14} \mathrm{~cm}^{-2}$ are strongly clustered in redshift, whereas all previous analyses have found that they are either unclustered (Sargent et al. 1980) or only very weakly clustered in redshift (e.g. Webb 1987; Barcons \& Webb 1991). How are these results compatible?

To examine this issue, we apply the standard method of measuring the Ly $\alpha$ TPCF to models of the Ly $\alpha$ absorption lines observed by CSKH. We first generate a set of Ly $\alpha$ absorption lines according to the results in Table 1a of CSKH. We adopt a constant CIV/HI ratio of $3 \times 10^{-3}$ and assume that the Doppler parameters are due to thermal motions, convolve the synthetic absorption lines with the appropriate instrumental response and add noise to match the actual signal-to-noise ratio of the observations. Next, we fit the resulting synthetic spectra using the Voigt profile fitting routine described previously by Lanzetta \& Bowen (1992). For each absorption line we add velocity components until the decrease in $\chi^{2}$ is smaller than the accompanying decrease in degrees of freedom, $\nu$. Finally, we construct the Ly $\alpha$ TPCF according to the procedures described in the previous section, but this time using the fitted redshifts instead of the actual redshifts.

The results are shown in Figure 1, which plots in the lower panel the high-redshift Ly $\alpha$ TPCF as traced by Ly $\alpha$ absorption lines. It is clear that the Ly $\alpha$ absorption lines cannot reveal the strong clustering indicated by the CIV absorption lines. The lower panel of Figure 1 may be directly compared with the high-redshift Ly $\alpha$ TPCF presented by Hu et al. (1995); both use observations of nearly the same quality, and both obtain practically identical results. Note that our stopping criterion for velocity components, $\Delta \chi^{2}<\Delta \nu$ purposely allows even marginally significant lines to be included. If no correlation is obtained even with this generous criterion, it certainly will not be found with a more conservative one. We therefore conclude that previous measurements of the high-redshift Ly $\alpha$ TPCF have underestimated the actual clustering of the absorbers due to unresolved blending of overlapping velocity components. 


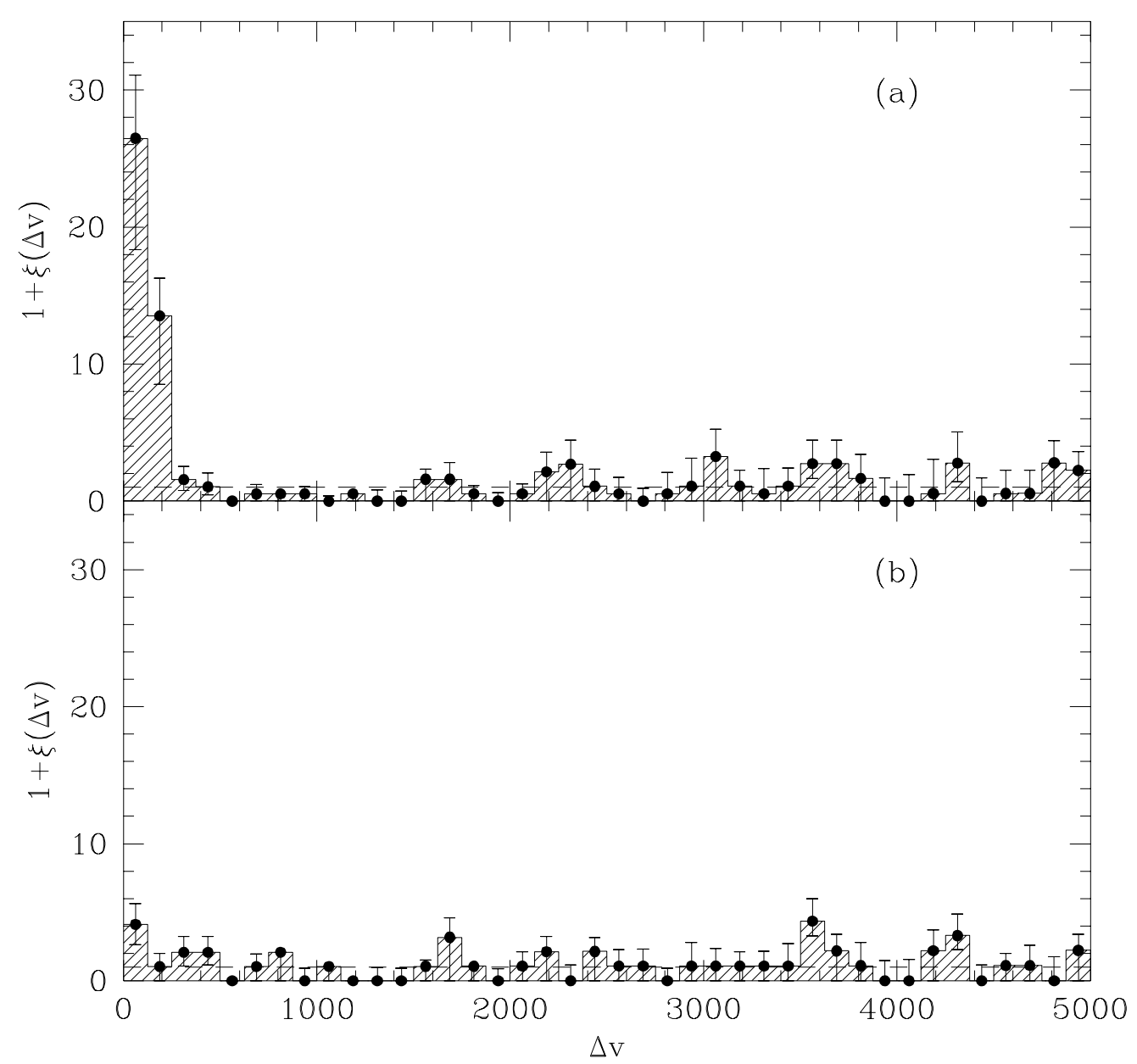

Fig. 1.- High-redshift Ly $\alpha$ TPCF as traced by very weak CIV absorption lines (upper panel) and as traced by Ly $\alpha$ absorption lines (lower panel). 


\subsection{Extension to Lower Column Densities}

The results of the previous section demonstrate that previous measurements of the high-redshift Ly $\alpha$ TPCF of absorbers with $N(\mathrm{H} \mathrm{I})>3 \times 10^{14} \mathrm{~cm}^{-2}$ have underestimated the actual clustering of the absorbers. Can this result extend to lower column densities for which blending is presumably weaker?

To examine this issue, we repeat the analysis described in the previous section for Ly $\alpha$ lines generated in two different ways. For the first simulation we assume that the CIV Doppler parameter $b$ is entirely due to thermal motion, so $b(H I)=\sqrt{12} b(C I V)$, and reduce the HI column densities by a factor of 100 with respect to the original ones. An example of just how a Voigt profile fit to high spectral resolution, high signal-to-noise ratio observations can underestimate the actual number of velocity components comprising an absorption line is shown in Figure 2, in which panel (a) shows the result of synthesizing the complex of lines at $z=2.7853$ toward Q0302-003 (with HI column densities decreased by a factor of 100 with respect to the original ones), panel (b) shows the actual components of the Ly $\alpha$ absorption line, and panel (c) shows the result of the Voigt profile fitting procedure. This complex of nine lines is adequately fitted $\left(\chi^{2} / \nu=0.91\right)$ with only three velocity components. The derived spectrum is then fitted in the same way used in $\S 3.2$. The resulting TPCF, Figure 3a, is still weaker than that of the CIV lines, but clearly detectable.

The assumption that all the velocity dispersion is thermal leads to temperatures in excess of $6 \times 10^{4} \mathrm{~K}$ in some cases, and this is inappropriate in most models (see Charlton, 1995, for a review of the models). We therefore add a second simulation in which the temperature is assumed to be $2 \times 10^{4} \mathrm{~K}$, and any excess Doppler parameter is ascribed to turbulence and applied equally to the CIV and Ly $\alpha$ lines. In a few cases the CIV Doppler parameter is just below the assumed thermal value, and in these cases we simply adopt the $2 \times 10^{4}$ thermal width for Ly $\alpha$. In this simulations the HI column density is assumed to be $10 \times$ that for CIV for each component. The Ly $\alpha$ lines are now generally narrower than in the first simulation, and so the component structure is more easily detected. Consequently, the Ly $\alpha$ line TPCF will have larger values at low velocity separations, as can be seen from Figure 3 (b). This should be compared with the observational result that little clustering is found at these redshifts (e.g. Rauch et al. , 1992).

These simulations are indeed too naïve, as we are not taking into account the increment in line number density at low column densities. This increment will produce strong blending effects among low column density lines themselves and also with the higher column density lines. Their combined effect is very difficult to simulate, as it depends very strongly on the higher-order correlation functions of the distribution of the lines. In addition, there are observations that suggest that the amplitude of the clustering is smaller at these low 


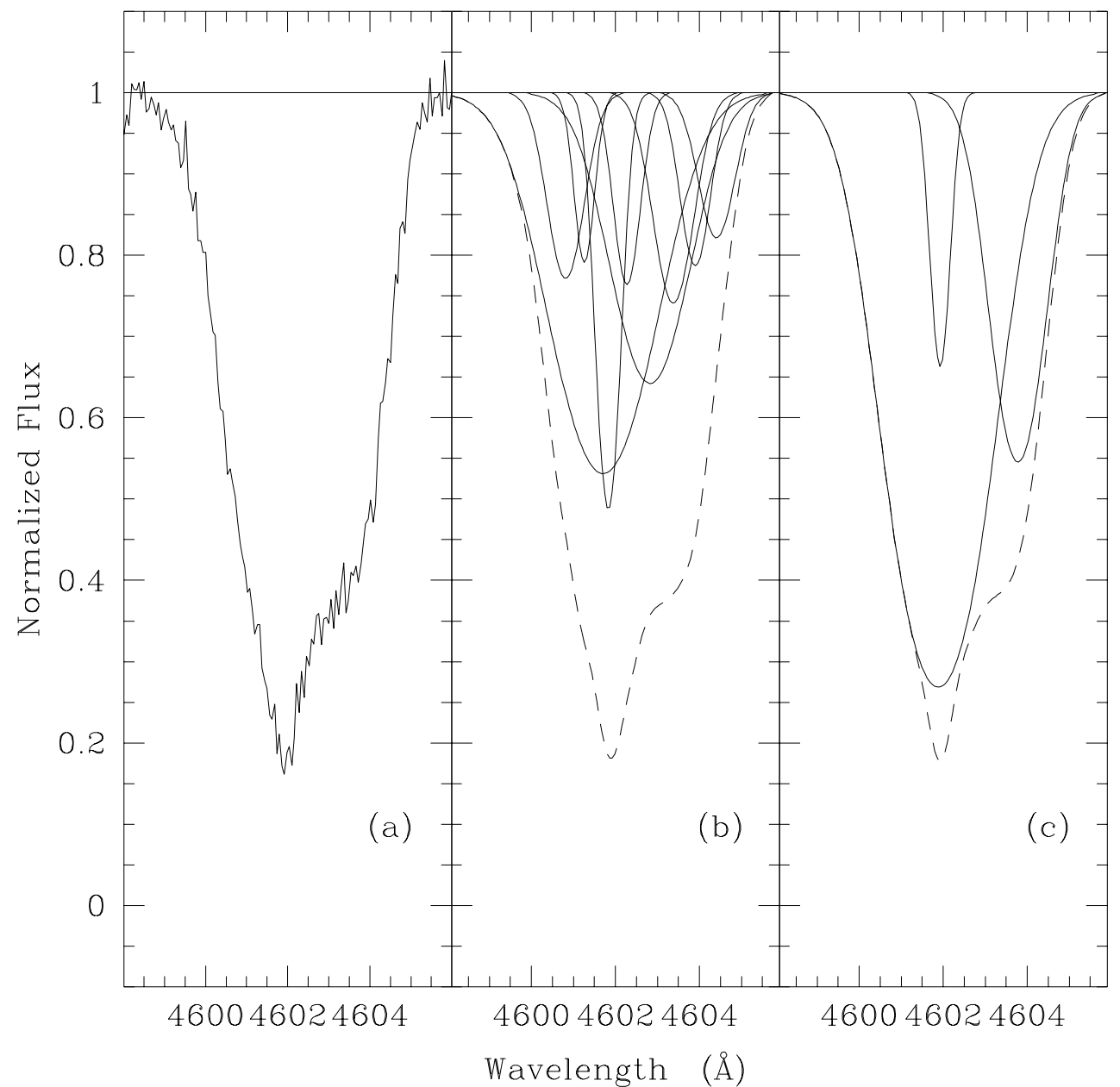

Fig. 2.- Example of how a Voigt profile fit to high spectral resolution, high signal-to-noise ratio observations can underestimate the actual number of velocity components comprising an absorption line. Panel (a) shows the result of synthesizing the complex of lines at $z=2.7853$ toward Q0302-003 (with HI column densities decreased by a factor of 100 with respect to the original column densities), panel (b) shows the actual components that comprise the Ly $\alpha$ absorption line, and panel (c) shows the result of the Voigt profile fitting procedure. 
column densities (Hu et al. 1995). All of these effects could very well erase all the signal in the correlation function for low column density lines, and hence we cannot conclude anything on the behavior of these low column density lines other than it is compatible with being clustered but maybe with a smaller clustering amplitude.

\section{Discussion and Summary}

The most significant result of the previous sections is that high-redshift Ly $\alpha$ absorbers with $N(\mathrm{H} \mathrm{I})>3 \times 10^{14} \mathrm{~cm}^{-2}$ are strongly clustered in redshift on velocity scales $\lesssim 250 \mathrm{~km} \mathrm{~s}^{-1}$. While the effect might be due to pairs of clouds with small velocity differences causing the observed TPCF (Miralda-Escudé et al. 1995; Rauch 1995), we could be seeing real clustering. With the observed velocity correlation length we can not decide whether the Ly $\alpha$ absorbers are independent entities, as has generally been assumed so far, or clouds within the halos of galaxies, the possibility we are exploring here. More detailed questions are even harder to answer, for example the type of galaxies in which the absorbers might reside, whether we are observing multiple clouds within the same galaxies, and the ionization state of carbon in the clouds. We can only ask if the strength of Ly $\alpha$ clustering is consistent with expectations of galaxy clustering at these early epochs.

To examine this issue, we consider a simple model for the evolution of the galaxy TPCF. In a first step, we ignore peculiar motions and motions of clouds within galaxies and assume that as a function of velocity and redshift the galaxy TPCF can be described by (Efstathiou et al. 1991)

$$
\xi(v, z)=(1+z)^{-3-\epsilon}\left[\frac{v}{r_{0} H(z)}\right]^{-1.8}
$$

where $H(z)$ is the Hubble constant at epoch $z$ (we take $q_{0}=0.5$ ) and $H_{0} r_{0}=550 \mathrm{~km} \mathrm{~s}^{-1}$ is the present-day galaxy correlation length. The evolutionary parameter $\epsilon$ takes the value -1.2 for comoving structures, 0 for virialized clusters, and 0.8 for linearly growing perturbations. Recent theoretical studies (Hamilton et al. 1991; Jain et al. 1995) show a steeper dependence on redshift at intermediate stages between the linear and virialized limits.

To avoid the divergence of this function at small values of $v$, we take $\xi(v, z)$ to be constant below a given velocity difference $v_{0}$ and equal to $\xi\left(v_{0}, z\right)$. We also convolve it with a Gaussian distribution with width $\sigma$ to account for random motions. In this way we get a set of different models defined by three parameters, $\epsilon, \sigma$, and $v_{0}$, which we allow to vary within the limits: $-2<\epsilon<4,0<\sigma<500 \mathrm{~km} \mathrm{~s}^{-1}$, and $1<v_{0}<80 \mathrm{~km} \mathrm{~s}^{-1}$. 


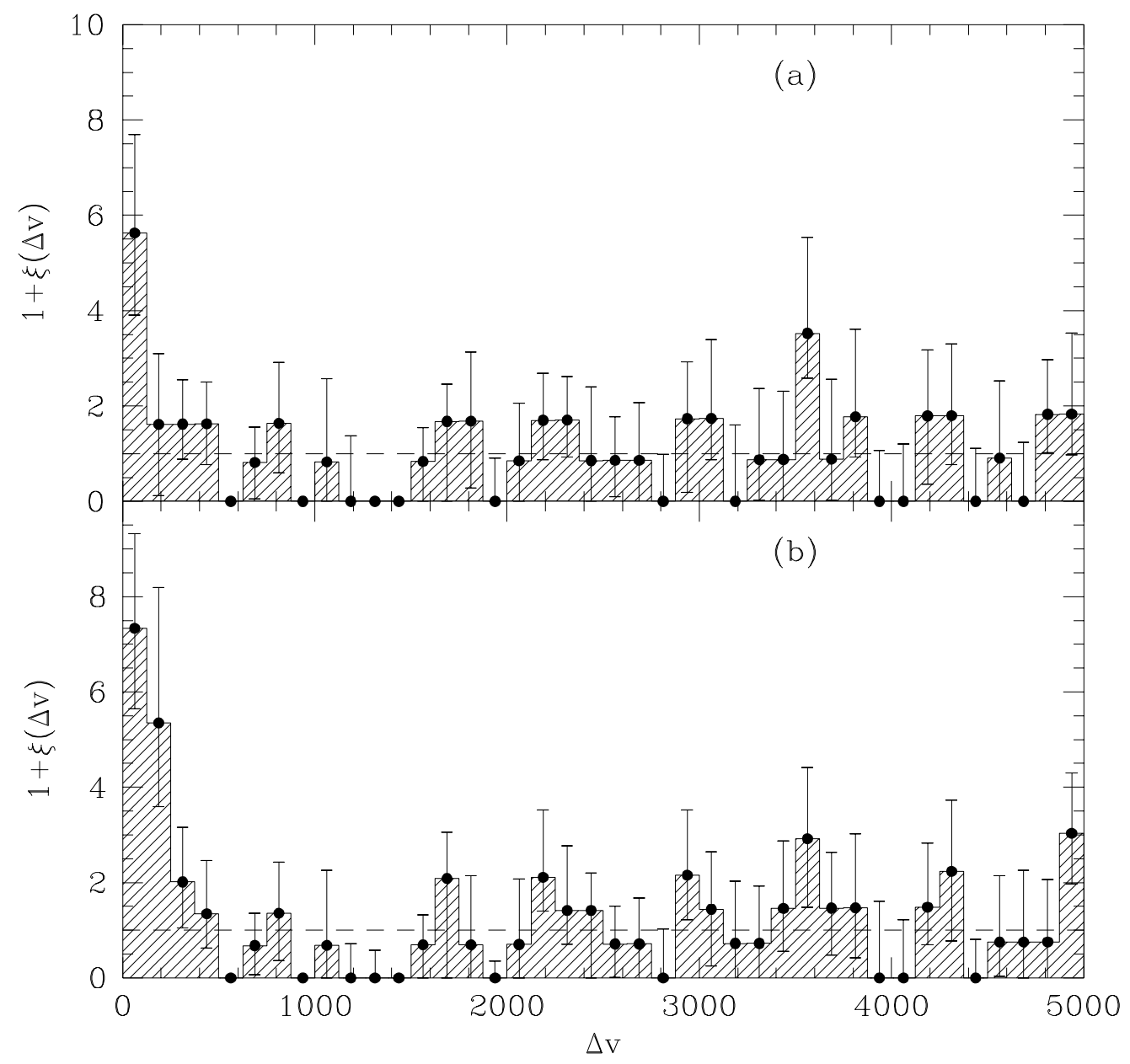

Fig. 3.- The TPCF for low column density clouds as obtained with two different models: Thermal-only broadening (panel a) and thermal plus turbulence (panel b). 
Predictions of all these models are then compared with the observed TPCF of high-redshift Ly $\alpha$ absorbers. The best fit is achieved for $\epsilon=2.4$ and $\sigma=100 \mathrm{~km} \mathrm{~s}^{-1}$. The 1-, 2- and 3- $\sigma$ confidence regions obtained using $v_{0}$ as an uninteresting parameter are plotted in Figure 4. From this calculation it is clear that if normal galaxies host the Ly $\alpha$ absorbers, at an average rate of one per galaxy, their correlation function is evolving rapidly and the combined intragalactic and intergalactic velocity dispersion is $\lesssim 150 \mathrm{~km} \mathrm{~s}^{-1}$.

Note that our redshift range is relatively small, $\Delta z \approx 0.6$, so that we can not separately fit $r_{0}$ and $\epsilon$. Our determination of $\epsilon$ is therefore anchored by the general galaxy correlation function at the present epoch. This may be inappropriate in several respects. We overestimate the correlation function if the host galaxies of the Ly $\alpha$ clouds are less clustered at the present epoch, for example if they are mostly spiral galaxies, or we underestimate it if there are multiple Ly $\alpha$ absorbers in galaxies at high redshift. The best we can deduce from our simple analysis is that the observed clustering of high-redshift Ly $\alpha$ absorbers is broadly consistent with the expected clustering of galaxies.

We conclude that (1) High-redshift Ly $\alpha$ absorbers with $N(\mathrm{H} \mathrm{I})>3 \times 10^{14} \mathrm{~cm}^{-2}$ are strongly clustered in redshift on velocity scales $\lesssim 250 \mathrm{~km} \mathrm{~s}^{-1}$, (2) Previous measurements of the Ly $\alpha$ TPCF have underestimated the actual clustering of the absorbers due to unresolved blending of overlapping velocity components, (3) The present observations may be consistent with the hypothesis that clustering of Ly $\alpha$ absorption systems persists to lower column densities, being likely that the clustering is smaller at low column densities, and (4) The observed TPCF is broadly compatible with that expected from galaxies at $z \sim 2-3$.

We interpret these results to suggest that many or most Ly $\alpha$ absorbers may arise in galaxies at all epochs, and therefore that the Ly $\alpha$ forest probes the processes of galaxy formation and evolution for redshifts $z \lesssim 5$.

AFS thanks E. Martínez-González for useful discussions and an anonymous referee for calling our attention on the gravitational collapse model. AFS acknowledges support provided by a Spanish MEC studentship. Partial financial support to AFS and XB is provided by the Spanish DGICYT under project PB92-0741, KML is supported by NASA grant NAGW-4433 and by a Career Development Award from the Dudley Observatory, and AY by NASA grant NAG-51228. 


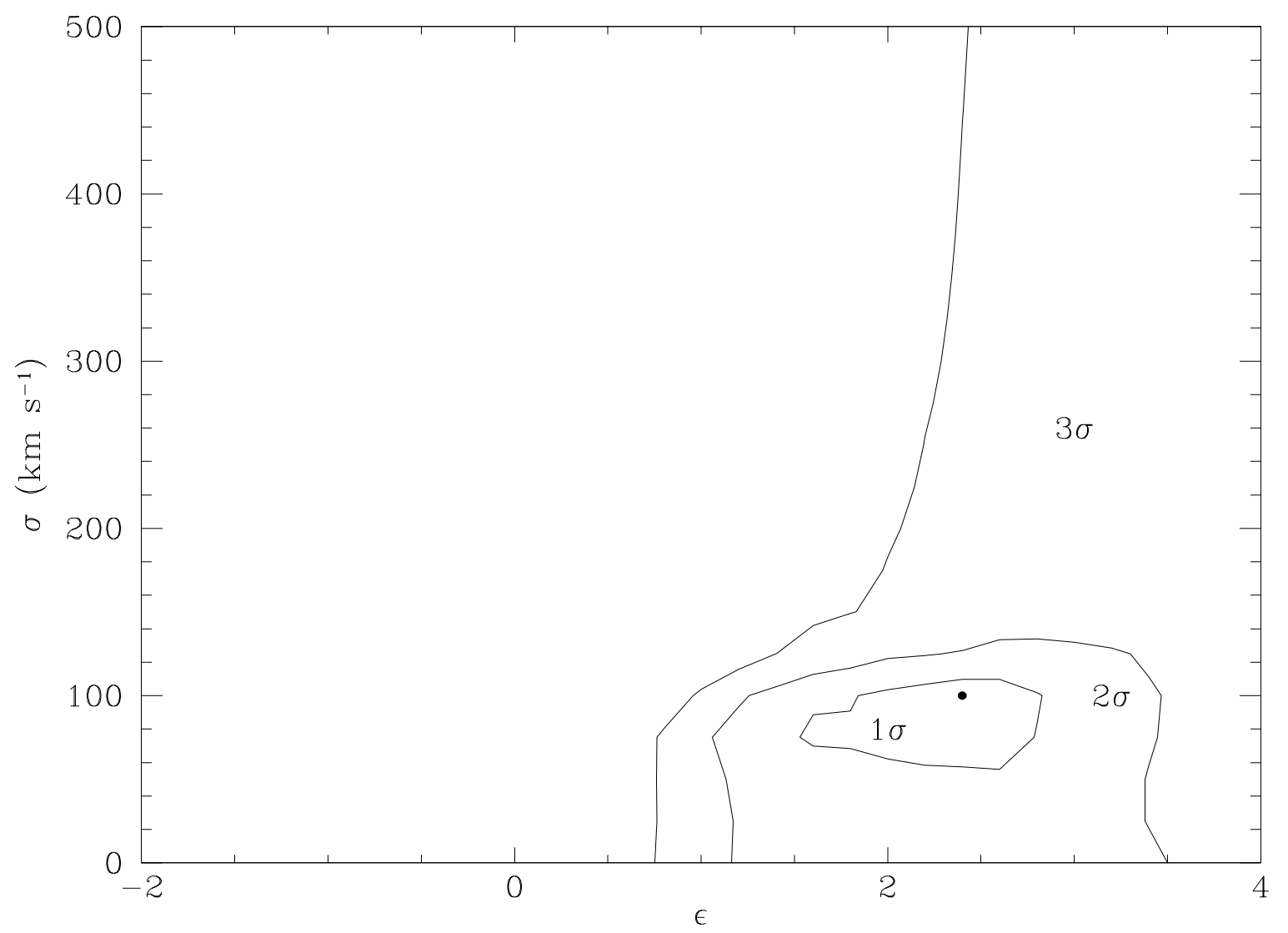

Fig. 4.- Confidence limits in the parameter space formed by the clustering evolution parameter $\epsilon$ and the typical velocity of galactic halo motions $\sigma$. 1-,2- and $3 \sigma$ confidence contours are plotted. 


\section{REFERENCES}

Bahcall, J. et al. 1995, ApJ, submitted

Barcons, X. \& Webb, J. K. 1991, MNRAS, 253, 207

Charlton, J. C. 1995, in QSO Absorption Lines, ed. G. Meylan (Berlin: Springer-Verlag), 405

Cowie, L. L., Songaila, A., Kim, T.-S., \& Hu, E. M. 1995, AJ, 109, 1522

Efstathiou, G., Bernstein, G., Katz, N., Tyson, J. A., \& Guhathakurta, P. 1991, ApJ, 380, L47

Hamilton, A. J. S., Kumar, P., Lu, E., \& Matthews, A. 1991, ApJ. 374, L1

Hu, E. M., Kim, T. S., Cowie, L. L., Songaila, A., \& Rauch, M. 1995, AJ, in press

Jain, B., Mo, H. J., \& White, S. D. M. 1995, MNRAS 276, L25

Lanzetta, K. M., \& Bowen, D. V. 1992, ApJ, 391, 48

Lanzetta, K. M., Bowen, D. V., Tytler, D., \& Webb, J. K. 1995, ApJ, 442, 538

Miralda-Escudé, J., Cen, R., Ostriker, J. P., \& Rauch, M. 1995, ApJ, submitted

Rauch, M. 1995, to appear in the proceedings of "Cold gas at High Redshift", eds. Bremer et al. (Hoogeeven, Holland)

Rauch, M., Carswell, R. F., Chaffee, F. H., Foltz, C. B., Webb, J. K., Weymann, R. J., Bechtold, J., \& Green, R. F. 1992, ApJ, 390, 387

Sargent, W. L. W., Young, P. J., Boksenberg, A., \& Tytler, D. 1980, ApJS, 42, 41

Webb, J. K. 1987, in Observational Cosmology, ed. A. Hewitt, G. Burbidge, \& L. Z. Fang (Dordrecht, Holland: Reidel/Kluwer Academic Press), 803 